\title{
AUMENTO DAS CARGAS DE TRABALHO EM TÉCNICOS DE ENFERMAGEM NA ATENÇÃO PRIMÁRIA À SAÚDE NO BRASIL
}

INCREASED WORKLOADS FOR NURSE TECHNICIANS IN PRIMARY HEALTH CARE IN BRAZIL

\author{
AUMENTO DE LAS CARGAS DE TRABAJO EN TÉCNICOS DE ENFERMERÍA EN LA ATENCIÓN \\ PRIMARIA A LA SALUD EN BRASIL
}

Magda Duarte dos Anjos Scherer ${ }^{1}$

Neura Angélica de Oliveira ${ }^{2}$

Denise Elvira Pires de Pires ${ }^{3}$
Letícia de Lima Trindade ${ }^{4}$

Ana Sofia Resque Gonçalves ${ }^{5}$

Monica Vieira ${ }^{6}$
Resumo Este artigo analisa os principais aspectos que contribuem para aumentar as cargas de trabalho de técnicos de enfermagem que atuam na atenção primária à saúde no Brasil, identifica o tipo de cargas e classifica os aspectos mais prevalentes para esse grupo ocupacional. Baseia-se em estudo multicêntrico realizado com 24 profissionais das regiões Sul, Centro-Oeste e Norte do Brasil, de 2011 a 2013, por meio de entrevistas semiestruturadas. Os dados foram sistematizados no software Atlas.ti e submetidos à análise temática. Os aspectos que mais contribuem para aumentar as cargas de trabalho são a baixa remuneração mensal, falta de recursos materiais, sobrecarga de trabalho, desgaste e adoecimento, relação com o usuário, e escassez de recursos humanos, prevalecendo cargas psíquicas e fisiológicas. Adequação das condições e organização do trabalho se mostram primordiais para amenizar as cargas de trabalho no cenário da atenção primária à saúde, e para promover a saúde, o bem-estar e a qualidade de vida no ambiente de trabalho dos técnicos de enfermagem.

Palavras-chave cargas de trabalho; saúde do trabalhador; técnico de enfermagem; atenção primária à saúde.

\begin{abstract}
In this article we analyze the main aspects that contribute to increase the workloads of nurse technicians working in primary health care in Brazil, we identify the type of loads and rank the most prevalent aspects of this occupational group. It is based on a multicenter study with 24 professionals from the South, Center-west and North regions of Brazil, from 2011 to 2013, using data from semi-structured interviews. The data were systematized in Atlas.ti software and submitted to thematic analysis. The aspects that contribute to increasing workloads are low monthly remuneration, lack of material resources, work overload, wear and illness, relationship with the user, and lack of human resources, prevailing psychological and physiological loads. Adequacy of the conditions and organization of work are shown paramount to ease the workload on the primary health care setting, and to promote the health, wellbeing and quality of life in the nurse technicians working environment.
\end{abstract}

Keywords work load; worker's health; nurse technician; primary health care. 


\section{Introdução}

Na atualidade vê-se o aumento de estudos que investigam as relações entre saúde e trabalho tendo em vista compreender a natureza desses processos e suas implicações na vida das pessoas (Cruz et al., 2010). Retoma-se, nesse contexto, que o trabalho humano nunca é neutro em relação à saúde dos indivíduos, favorecendo seja a saúde ou a doença, uma vez que pode ser gerador de diversos sentimentos, ora de prazer, ora de sofrimento (Dejours, Abdoucheli e Jayet, 2011).

Na atenção primária à saúde (APS), os trabalhadores manifestam satisfação quando há afinidade com o trabalho e com a profissão, quando gostam do que fazem (Lima et al., 2014). Contudo, de modo geral, o trabalho tem se caracterizado, de um lado, por limitações estruturais nos serviços, falta de recursos humanos, formação inadequada, carência de recursos materiais e hierarquização entre os profissionais que compõem as equipes, bem como pela relação desigual entre trabalhadores e usuários. Trabalhar na APS exige a construção de vínculos entre os profissionais e deles com os usuários, capacidade para lidar com a complexidade do processo saúde-doença e a articulação de práticas e saberes que ultrapassam o núcleo de competência profissional. Esses são fatores responsáveis pela elevação das cargas de trabalho sobre os profissionais e que, ao não serem reconhecidos e enfrentados, podem limitar o alcance das ações de promoção e integralidade na APS (Scherer, Pires e Soratto, 2014).

As cargas de trabalho são elementos do processo de trabalho que interagem entre si e com o corpo do trabalhador, desencadeando alterações nos processos biopsíquicos que se manifestam em desgastes físicos e psíquicos potenciais ou efetivamente apresentados. Para compreender as cargas, é preciso levar em conta três dimensões de análise: as ações do trabalhador, o contexto em que elas ocorrem e as consequências geradas por essas ações (Laurell e Noriega, 1989).

As cargas de trabalho podem ser agrupadas, segundo a sua natureza ou características básicas, naquelas que têm materialidade externa e que se modificam na interação com o organismo (físicas, químicas, biológicas e mecânicas) e naquelas que adquirem materialidade no próprio corpo humano e se expressam internamente por meio dele (fisiológicas e psíquicas). Assim, as cargas de trabalho são decompostas em tipos específicos que compreendem os riscos particulares; o que não quer dizer que elas se constituam da soma dos riscos, porque adquirem significado a partir da dinâmica global do processo de trabalho (Laurell e Noriega, 1989).

Estudos em diferentes cenários assistenciais assinalam diversos fatores que determinam o aumento das cargas de trabalho, principalmente as cargas psíquicas, tais como: a organização do trabalho; o excesso de atribuições 
e demandas; a insuficiência e a má qualidade dos recursos materiais e os baixos investimentos financeiros; os problemas de relacionamento com a equipe, usuários e famílias; e a desvalorização dos profissionais por parte dos usuários e chefias, dentre outros (Trindade e Pires, 2013; Martins et al., 2013).

O trabalho de enfermagem é reconhecido como deletério, árduo e arriscado para os profissionais, sendo um local propício para o adoecimento, com exposição a acidentes e agravos de ordem fisiológica (Elias e Navarro, 2006), que, somados às precárias condições de trabalho, podem levar a transtornos mentais, como ansiedade e depressão (Schmoeller et al., 2011).

Os salários baixos, a dupla jornada, o aumento da carga horária, a distância entre o trabalho prescrito e o realizado, as situações de tensão nos serviços de saúde, tanto pelo tipo de atenção aos indivíduos em momentos críticos quanto pela fragmentação do trabalho, a hierarquização e a desvalorização social dentro da equipe, entre outros fatores, juntam-se às condições de trabalho da equipe de enfermagem e interferem na qualidade do cuidado ao usuário e no desgaste mental, aumentando as cargas de trabalho dos profissionais (Schmoeller et al., 2011), levando à abdicação da profissão e diminuindo os recursos humanos na enfermagem (Medeiros et al., 2006).

Dos profissionais da enfermagem inscritos nos conselhos regionais, os enfermeiros compõem $17,9 \%$ da força de trabalho, enquanto os técnicos representam $50 \%$, o que lhes confere papel fundamental no cumprimento das metas institucionais (Conselho Regional de Enfermagem da Bahia, 2014).

Nesse contexto, este artigo analisa os principais aspectos que contribuem para aumentar as cargas de trabalho dos técnicos de enfermagem que atuam na atenção primária à saúde no Brasil, identifica o tipo de cargas e classifica os aspectos mais prevalentes para esse grupo ocupacional.

\section{Método}

Trata-se de um estudo descritivo multicêntrico nacional com abordagem qualitativa, desenvolvido em três regiões do país, com equipes da APS, sendo para este artigo apresentados os achados referentes aos técnicos de enfermagem.

Participaram 23 técnicos de enfermagem: cinco de quatro unidades de saúde da Região Sul; dez de três unidades da Região Norte; e oito de cinco unidades de saúde da Região Centro-Oeste, os quais compunham equipes de serviços considerados de boa qualidade segundo informações das gerências das diferentes regiões investigadas incluídas no estudo.

Os dados foram coletados de novembro de 2010 a fevereiro de 2013 por meio de entrevistas semiestruturadas, cujo número foi definido pelo critério de saturação dos dados. As entrevistas foram guiadas por roteiro que buscava identificar e caracterizar informações relativas ao trabalho dos profissionais 
de saúde das equipes, a estrutura organizacional da instituição, a composição das equipes, as práticas assistenciais, a organização e a divisão do trabalho e o modelo assistencial e sua influência na saúde, na satisfação e nas cargas de trabalho dos profissionais de saúde. Neste artigo, o foco foram os aspectos que elevavam as cargas de trabalho.

O trabalho de campo foi precedido de autorização das secretarias municipais de saúde; sensibilização dos profissionais para participação na pesquisa; capacitação dos pesquisadores para o trabalho de campo; e teste do roteiro de entrevistas.

Os resultados foram submetidos à análise temática de conteúdo, com o auxílio do software Atlas.ti 7.0 (Qualitative Research and Solutions), que contribuiu para a triangulação analítica dos dados de cada região.

Para garantir o anonimato dos participantes, as falas foram identificadas por uma sequência de letras que significam, respectivamente, TE para técnico de enfermagem, seguido do número cardinal de ordem da entrevista e a região.

O projeto foi aprovado no Comitê de Ética em Pesquisa com Seres Humanos da Universidade Federal de Santa Catarina, parecer n. 971/2011, e, ainda, pelo Comitê de Ética em Pesquisa da Fundação de Ensino e Pesquisas em Ciências da Saúde do Governo do Distrito Federal (CAAE n. 58/2012). Todos os participantes assinaram o termo de consentimento livre e esclarecido.

\section{Resultados e discussão}

A análise dos dados demonstrou que os principais aspectos que aumentam as cargas entre os técnicos de enfermagem na APS são a baixa remuneração mensal, a falta de recursos materiais para assistência, a sobrecarga de trabalho, o sofrimento físico (adoecimento), a relação estabelecida com o usuário e a escassez de recursos humanos, sinalizando a presença de cargas fisiológicas e psíquicas, conforme o Quadro 1.

Quadro 1

\begin{tabular}{|c|c|c|c|}
\hline \multicolumn{4}{|c|}{ Perfil dos docentes participantes da pesquisa. Florianópolis, 2013} \\
\hline Ordem & Fontes de aumento das cargas de trabalho (categorias) & n. de participantes & Tipo(s) de cargas \\
\hline 1 & Baixa remuneração & 14 & Psíquicas \\
\hline 2 & Falta de recursos materiais & 13 & Fisiológicas e psíquicas \\
\hline 3 & Sobrecarga de trabalho & 12 & Fisiológicas e psíquicas \\
\hline 4 & Escassez de recursos humanos & 12 & Fisiológicas e psíquicas \\
\hline 5 & Desgaste e adoecimento dos profissionais & 10 & Fisiológicas e psíquicas \\
\hline 6 & Relação profissional/usuário & 10 & Fisiológicas e psíquicas \\
\hline 7 & Escassez de recursos humanos & 9 & Fisiológicas e psíquicas \\
\hline
\end{tabular}

Fonte: Os autores. 


\section{Baixa remuneração}

O descontentamento com a remuneração mensal é o aspecto que mais aumenta as cargas de trabalho dos que atuam nas três regiões avaliadas. Os entrevistados também estão insatisfeitos com os auxílios creche e alimentação, e abordam a diferença salarial em relação a outras categorias funcionais, que são mais bem pagas do que os profissionais de saúde. A falta de plano de carreira para os profissionais e a necessidade de outro vínculo empregatício para aumentar a renda é um dos aspectos que eleva a sobrecarga de trabalho:

(...) pode melhorar alguns auxílios... Outras categorias do governo recebem auxílios melhores que os nossos, e como a saúde é uma área muito importante... Eu acho que não deveria haver desigualdade... (TE27 Centro-Oeste).

(...) não tem plano de carreira para a enfermagem... A carga horária de oito horas não satisfaz, seria melhor seis horas, também para o usuário: tu pegarias aquele povo que não está [sendo atendido], porque muitas famílias deixam de ser acompanhadas por causa dos horários (TE80 Sul).

Com o salário não, com certeza não estou satisfeita com o meu salário (TE48 Norte).

O salário também é muito baixo, por isso mesmo que eu tenho que trabalhar em dois empregos... (TE70 Sul).

Essa situação se assemelha aos resultados encontrados em estudo realizado em 15 municípios no Sul do Brasil, em 53 equipes da Estratégia Saúde da Família (ESF): todos os profissionais trabalhavam 40 horas semanais e $54,54 \%$ tinham outro emprego. O segundo vínculo era justificado pela insatisfação com o salário, necessidade de complementar a renda familiar e pela busca de melhoria do nível de inserção social (Trindade e Pires, 2013).

Percebem-se grandes diferenças salariais de acordo com os municípios em que o profissional atua, principalmente em decorrência da forma de contratação e vínculo com a instituição. No Distrito Federal, a Secretaria de Estado de Saúde comumente contrata seus profissionais por meio de concurso público, e eles são contratados como estatutários e são mais bem remunerados que os técnicos de enfermagem das regiões Norte e Sul (Moraes, 2013). No estudo de Cotta et al. (2006) sobre satisfação salarial com profissionais da estratégia saúde da família: médicos, enfermeiros, auxiliares de enfermagem e agentes comunitários de saúde, 50\% dos entrevistados se encontravam insatisfeitos com a remuneração, e os resultados foram relacionados à contratação por outros meios, que não o concurso público. 
As condições do contrato para o trabalho na APS dependem muito do município, não são raros os exemplos de contratação instável e falta de incentivos para a dedicação exclusiva (Santini et al., 2010). Esse cenário pode dificultar a criação de vínculo do trabalhador com a APS e fazê-lo recorrer às jornadas duplas, gerando insatisfação e adoecimentos, e influenciando na qualidade da assistência (Trindade e Pires, 2013). Cabe salientar que o auxílio alimentação e a disponibilidade de creches para os filhos dos profissionais se destacam como direitos trabalhistas que favorecem a permanência dos trabalhadores no cenário de trabalho e minimizam a defasagem dos salários dos profissionais.

\section{Falta de recursos materiais}

A falta de recursos materiais para o trabalho na APS é a segunda maior queixa dos entrevistados. Foi mencionada como fator de desmotivação e aumento das cargas no trabalho. Os participantes relataram dificuldade para prestar assistência à saúde compatível com o conhecimento técnico de enfermagem, que exige deles o uso correto de determinados instrumentais e insumos para que um agravo evolua bem e não seja piorado pelo próprio procedimento. Isso pode acontecer caso o procedimento não seja realizado dentro de normas e padrões de assepsia e com a cobertura adequada, como no caso de curativos.

Os profissionais também demonstram preocupação com o usuário que busca o atendimento para fazer exames preventivos que podem revelar um problema mais grave precocemente, ou que deva ser tratado imediatamente, mas tem que voltar outro dia por falta de materiais básicos para a sua execução. Muitas vezes os profissionais usam o próprio dinheiro para comprar equipamentos, tais como aparelhos para aferição da pressão arterial, que é um marcador básico em ações programáticas.

Outro problema enfrentado é a falta de estrutura física adequada para o acolhimento dos usuários. As falas demonstram insatisfação com essa realidade, agravada na medida em que há dificuldade de comunicação com as chefias, fazendo o profissional sentir-se desvalorizado em suas demandas e saberes:

(...) está faltando material. Muitas vezes você tem que improvisar... Não tem pinça porque a autoclave está quebrada. Falta material para curativo (...), mas o paciente não quer nem saber, ele está ali na sua frente. Isso é frustrante (TE14 Centro-Oeste).

(...) os funcionários precisam de mais atenção também... Às vezes a gente não tem nem como trabalhar. Aquele estetoscópio é meu, eu comprei com o meu dinheiro. 
Às vezes a gente reclama [com a chefia] que está atrapalhado e, assim, a gente não vê muito interesse [por parte dela] (TE24 Centro-Oeste).

Não tem material, sala de vacina, fico desmotivada (TE51 Norte).

(...) a estrutura física da unidade, porque é tudo muito improvisado, é parte de uma escola... Por falta de material para coleta de preventivo, às vezes tem que mandar o paciente voltar, então isso dificulta um pouco... (TE81 Sul).

Constata-se que as dificuldades referentes à estrutura podem afetar o atendimento às famílias e interferem na qualidade da assistência. Mas os serviços de saúde devem oferecer condições sociopolíticas, humanas e materiais que auxiliem a produção de um trabalho com qualidade para quem atua e para quem recebe a assistência (Oliveira, Carvalho e Silva, 2008; Trindade e Pires, 2013).

As limitações na estrutura das unidades de saúde e na rede de serviços resultam em sofrimento dos profissionais e influenciam na qualidade dos processos de trabalho, mas, mesmo diante das precárias condições, segundo Bertoncini, Pires e Scherer (2011), os profissionais buscam alternativas para serem eficazes com base nos valores de direito à saúde, acesso e qualidade.

\section{Sobrecarga de trabalho e escassez de recursos humanos}

As categorias sobrecarga de trabalho e escassez de recursos humanos nas unidades de saúde da APS representam, em conjunto, importantes aspectos que aumentam as cargas e interatuam entre si, e a falta de recursos humanos influencia diretamente no aumento da sobrecarga. Assim, optou-se por apresentar essas categorias de forma agrupada, segundo as relações que os participantes fizeram entre elas, independentemente da frequência com que apareceram.

O trabalho na APS exige dos técnicos de enfermagem uma diversidade de saberes para realizar as ações de promoção de saúde e prevenção de doenças, tanto no nível individual quanto no coletivo, na unidade em que trabalham e nos lares dos usuários, de acordo com os princípios e diretrizes do Sistema Único de Saúde (SUS). Por isso, são necessárias ferramentas cognitivas afiadas para desempenhar todas as funções demandadas em seu dia a dia com qualidade e sensibilidade, o que determina um maior 'uso de si'. Essa dedicação intensificada pode levar ao desgaste psíquico e fisiológico, principalmente por desempenhar suas atribuições num novo modelo de atenção, com uma nova proposta de cuidado em saúde, um novo paradigma, que propõe superar a hegemonia biomédica, focando numa atenção multi e interdisciplinar. 
Nesse sentido, o trabalho convoca a subjetividade do trabalhador para uma atenção humanizada ao usuário, observando não apenas suas queixas, mas também o seu contexto social e ambiental. Ao mesmo tempo em que devem adaptar-se ao novo modelo de atenção em curso na APS, assumindo responsabilidades do campo multiprofissional prescritas pela Política Nacional da Atenção Básica (PNAB), os técnicos de enfermagem são exigidos nas várias atribuições específicas do cargo, que se tornam mais extenuantes na medida em que não há recursos humanos suficientes, aumentando a sobrecarga desses trabalhadores.

Segundo os técnicos das três regiões, a rotina de trabalho é exaustiva, porque realizam múltiplas tarefas na jornada: triagem, anamnese inicial em cada usuário, preenchimento manual de várias fichas antes do encaminhamento do usuário para as consultas com outros profissionais, atendimento na farmácia para entregar as medicações e dar orientações sobre o seu uso, na sala de vacinas, colocação de curativos na unidade e a domicílio, visitas às famílias, dentre outros. A sobrecarrega é aumentada pela exigência de rápido atendimento. Consideram que dois técnicos de enfermagem por equipe é pouco diante das demandas. Quando esses profissionais se ausentam, seja por adoecimento ou por outros motivos, não há outros técnicos para substituí-los e recompor a equipe. Ao mesmo tempo, por saberem da dificuldade que se impõe quando um membro da equipe está ausente, eles mesmos se sentem mal por terem que se ausentar para cuidar da própria saúde.

A gente ainda corre o risco de cometer um erro porque, se eu estou triando, aí já tem uma fila para a farmácia. Então, eu chego na farmácia para entregar o remédio e já tem mais três [pacientes] esperando para triar, corre o risco de, na pressa, ao entregar uma medicação, entregar errado... (TE12 Centro Oeste).

Ter apenas dois técnicos de enfermagem numa equipe para atender uma unidade toda - onde tudo tem que funcionar, farmácia, curativo, medicação, triagem, guarda de material, guarda de prontuário, emergência, semi-internação, onde tudo isso é executado por dois técnicos de enfermagem --, sobrecarga fica imensa. É muito serviço para ser desenvolvido ao mesmo tempo... (TE14 Centro-Oeste).

Você chega para trabalhar, aí você vê: é fulano de atestado, quando você vê, só está um pouquinho de gente trabalhando... Eu acho também que, diante de tantos atestados, a gente tem que discutir o que está acontecendo... (TE25 Centro-Oeste).

Os funcionários são poucos para dar continuidade ao serviço e acabo passando do meu horário (TE57 Norte). 
Porque a minha equipe está incompleta, falta uma técnica, eu estou com um enfermeiro e um médico e eu estou sozinha... Dobra o trabalho, a gente não consegue fazer um trabalho como a gente quer por falta de um técnico... (TE81 Sul).

As dificuldades sentidas, relativas à grande demanda de trabalho e à pouca disponibilidade de recursos materiais e humanos, são bem características da área da saúde. O sofrimento decorrente deriva da necessidade de ajustar recursos finitos a necessidades de cuidados de saúde infinitas e crescentes por parte da população (Almeida e Pires, 2007).

A insuficiência de recursos humanos, gerando a sobrecarga de atribuições para a equipe de enfermagem, é fonte de múltiplos transtornos: exige um ritmo mais acelerado, aumenta a responsabilização por um volume muito maior de tarefas no processo do cuidado e repercute em desgaste. É preciso dimensionar o efetivo de pessoal, atendendo a aspectos como a relação entre os custos da saúde e efetividade assistencial; o grau de vínculo e dependência dos usuários; as cargas de trabalho na equipe de enfermagem; e os fatores que influenciam na qualidade do trabalho desses profissionais, entre outros (Magalhães, Riboldi e Dall'Agnoll, 2009).

Tanto a falta de trabalho quanto o excesso dele afetam a saúde do profissional. As condições de trabalho quando desfavoráveis comprometem a saúde do trabalhador, levando o indivíduo ao adoecimento (Dejours, Abdoucheli e Jayet, 2011). Porém o excesso de trabalho talvez seja a indicação mais precisa do desequilíbrio entre o trabalhador e o ambiente no qual trabalha, e, associado ao ritmo intenso, interfere na qualidade da assistência, nas relações com colegas e contribui para o desgaste físico e emocional do trabalhador e para o absenteísmo (Ritter, Stumm e Kircher, 2009; Girondi et al., 2010; Santana et al., 2013).

\section{Desgaste e adoecimento dos profissionais}

O sofrimento físico relacionado ao adoecimento é relatado por vários entrevistados como um fator de aumento das cargas de trabalho. Percebe-se nas falas grande desgaste do corpo e mente em decorrência da organização e dos modos de trabalhar. A alta demanda de trabalho, materiais pesados a transportar, a violência a que estão expostos, a ausência dos colegas adoecidos, a falta de entrosamento da equipe, problemas ligados ao contato com o usuário e até mesmo a falta de água potável nas unidades de saúde são aspectos encontrados nas falas dos entrevistados que podem causar ou aumentar o sofrimento dos trabalhadores, traduzindo-se por vezes em doenças. Os trechos das falas abaixo exemplificam as consequências do desgaste sobre a saúde dos participantes da pesquisa: 
(...) às vezes eu saio com taquicardia daqui, dependendo do tumulto que dá. Eu vejo colegas minhas aqui que nunca tinham passado um atestado e agora é um atrás do outro... (TE25 Centro-Oeste).

Tenho hipotireoidismo, problema hepático, ganhei peso e isso por conta da falta de exercício... (TE57 Norte).

Tenho três hérnias de disco, passei um tempo na Odontologia, então carregava material... (TE58 Norte).

Nestes dois últimos anos eu definhei, eu adoeci, eu não sei, assim, se é físico, ou se psíquico... Tu não tens vontade de ir para o teu emprego, porque tu chegas no teu trabalho, tu dizes um bom dia, e a pessoa nem te responde, nem olha para você, te nega até um bom dia, tu imaginas o que vem no restante do dia... (TE71 Sul).

(...) várias pessoas estão adoecendo, e mais da área de enfermagem. É porque os outros, eles não têm esse contato mais próximo com o paciente, mais é a enfermagem mesmo... Não estou falando só de técnico de enfermagem. É a enfermagem em geral (TE25 Sul).

A depender do tipo de tarefa, de sua complexidade e da diversidade, como é o caso das atividades desempenhadas na APS, os trabalhadores estão expostos a diferentes cargas de trabalho (Dilélio et al., 2012; Robazzi et al., 2012). O trabalho, muitas vezes em situação rotineira, mecânica e repetitiva, pode comprometer a saúde, gerando processos de desgaste físico os quais podem acarretar a perda ou a redução da capacidade de produzir, bem como o agravamento e o aparecimento de doenças como lesões osteoarticulares, dentre outras.

A exposição às cargas psíquicas está associada à supervisão estrita; ritmo acelerado; trabalho parcelado, monótono e repetitivo; dificuldades na comunicação; agressão psíquica; fadiga; tensão; estresse e insatisfação. Os profissionais de enfermagem são os mais acometidos por esses agravos, confirmando assim a grande exposição desses trabalhadores a processos que geram morbidade (Mininel, Baptista e Andres, 2011; Santana, 2013).

Uma revisão integrativa sobre cargas de trabalho e condições de trabalho da enfermagem encontrou vários artigos que ressaltam a ocorrência de frequentes acidentes com material perfurocortante ou fluídos e secreções corporais e de problemas de saúde como contusões, hipertensão arterial, alergias, epigastralgias, problemas musculoesqueléticos, adoecimento e sofrimento mental, entre outros, relacionados à sobrecarga pelo excesso de trabalho, mostrando-se também como a causa do afastamento do trabalho desses profissionais (Schmoeller et al., 2011). 


\section{Relação profissional/usuário}

Os embates com os usuários são exaustivos e desgastantes para os profissionais, considerados pelos participantes da pesquisa um dos principais causadores do aumento das cargas de trabalho.

Nas unidades, os técnicos de enfermagem são, na maioria das vezes, os que têm o primeiro contato com o usuário, deparando-se com indivíduos com problemas que nem sempre podem ser acolhidos pela equipe. Esse profissional, no cotidiano, convive com uma realidade organizacional que o limita para agir de acordo com o novo modelo de atenção preconizado na APS, pois o trabalho das equipes continua centrado no tratamento dos agravos e das doenças crônicas já instaladas. Essa maneira de cuidar colabora para o aumento das filas, e a negativa de uma consulta clínica é uma das maiores causas de conflitos entre os profissionais e os usuários.

O excesso de demanda e a complexidade da atenção requerem do profissional a realização de acolhimento humanizado e de qualidade, produzindo um diálogo gerador de vínculo com o usuário. Entretanto, segundo os participantes, a tentativa de convencimento do usuário à adesão ao novo modelo de atenção é muitas vezes um elemento que aumenta as cargas no trabalho.

As pessoas chegam aqui bem nervosas, dizendo que você é a culpada de tudo, chegam com receitas vencidas e querem ser atendidas, querem o remédio. Às vezes chegam aqui e o médico já não tem mais vaga... Já teve gente que quis avançar em mim... (TE16 Centro-Oeste).

Quando acontece alguma coisa ruim no serviço, os usuários denunciam, mas quando está acontecendo um atendimento bom, eficiente, ninguém ressalta isso... Falta um pouco de reconhecimento (TE27 Centro-Oeste).

A verdade é que essas visitas não são muito bem aceitas, tem gente que está dormindo, outros ocupados, alguns não gostam, acham que nossa visita não é tão importante (TE49 Norte).

(...) ela [a pessoa] nem toma o remédio que tem que tomar, chegam com aquele monte de medicamentos do mês passado, como os hipertensos... Tu fazes palestra todo mês, tu orientas, tu entregas o medicamento, a pessoa não segue o que tem que fazer, então (...) Tu auxilias, e a pessoa não faz o que tem que fazer e isso desgasta o profissional... (TE71 Sul).

A negativa ao atendimento das necessidades de saúde gera desconforto e até agressões aos profissionais. O difícil acesso, a estrutura física deficitária 
dos serviços de saúde, a deficiência de recursos humanos e o acolhimento ineficiente do usuário na APS representam falhas na qualidade da atenção à saúde e são possíveis geradores de conflitos. Segundo estudo de Gehring Júnior et al. (2007), a insatisfação dos usuários provoca aumento das cargas psíquicas quando se expressam em agressões como desrespeito, ofensas verbais, discriminação e humilhação, podendo contribuir para o desenvolvimento de quadros patológicos psiquiátricos, como síndrome do pânico ou mesmo doenças cardiovasculares (Mininel, Baptista e Andres, 2011). A melhoria das condições do serviço, o acesso facilitado ao usuário e uma equipe mais integrada foram aspectos levantados no estudo de Kaiser e Bianchi (2008) como elementos minimizadores das situações agressivas no contexto da APS.

\section{Considerações finais}

O trabalho na APS é muito complexo e responsável pelo aumento das cargas sobre os profissionais que nela atuam. Demanda maior dedicação e atenção por parte dos profissionais da enfermagem que lidam com subjetividades e têm que intensificar sua atuação todo o tempo, visto que, na maioria das vezes, são o primeiro contato do público com a atenção primária à saúde. O trabalho requer, assim, uma convivência mais próxima com a comunidade, um diálogo constante e acolhedor, que seja capaz de criar vínculo e conquistar a confiança dela.

Este estudo revelou que os principais aspectos que causam desgaste e contribuem para o aumento das cargas de trabalho dos técnicos de enfermagem na APS podem interferir na qualidade dos serviços ofertados. Tais aspectos influenciam o processo de sofrimento, adoecimento e absenteísmo desses profissionais. Conclui-se que os elementos que mais aumentam as cargas de trabalho, como a baixa remuneração, a falta de recursos humanos e a sobrecarga poderiam ser minimizados, em parte, com a formulação de políticas de gestão do trabalho e da educação que levem em conta a especificidade do trabalho na APS. Entre essas destacam-se políticas de cargos e salários mais igualitárias, investimento na formação e efetivação de profissionais qualificados para lidar com as dinâmicas envolvidas no trabalho na APS, processo de educação permanente e continuada com os trabalhadores e uma gestão que valorize o profissional e proporcione um ambiente de trabalho gratificante. Espera-se que assim esse trabalhador perceba que sua atuação é importante no processo de trabalho.

Na organização do trabalho sempre existirão elementos que podem aumentar as cargas de trabalho. Esses fatores são próprios da inserção do trabalhador num meio adverso, mas a tradução do contato com esses elementos 
em sofrimento ou adoecimento dependerá do que cada pessoa traz consigo, da sua própria subjetividade, da maneira como ela é capaz de se integrar com o meio e lidar com esses elementos. O que sensibiliza um certo indivíduo pode não ter a mesma força sobre outro, isso tem a ver com as histórias pessoais, com as percepções e representações vivenciadas de forma única pelos profissionais. Contudo, a adequação das condições e da organização do trabalho se mostram primordiais para amenizar as cargas de trabalho no cenário da APS e para a promoção da saúde, do bem-estar e da qualidade de vida no ambiente de trabalho do técnico de enfermagem.

Observou-se que os resultados apresentados neste trabalho correspondem em vários aspectos aos encontrados em outros estudos sobre os elementos que aumentam as cargas de trabalho, tanto os relacionados com a categoria profissional estudada quanto com outras categorias da área de saúde, demonstrando a necessidade de uma atenção mais humanizada e acolhedora por parte dos gestores aos trabalhadores da APS.

\section{Colaboradores}

Magda Duarte dos Anjos Scherer, Neura Angélica de Oliveira, Denise Elvira Pires de Pires e Letícia de Lima Trindade participaram da concepção, da análise dos dados, da redação e da aprovação da versão final do artigo. Ana Sofia Resque e Monica Vieira participaram da análise dos dados, da redação e da aprovação da versão final do manuscrito. 
Resumen Este artículo analiza los principales aspectos que contribuyen para aumentar las cargas de trabajo de técnicos de enfermería que actúan en la atención primaria a la salud en Brasil, identifica el tipo de cargas y clasifica los aspectos más prevalentes para ese grupo ocupacional. Es basado en estudio multicéntrico realizado con 24 profesionales de las regiones Sur, Centro-Oeste y Norte de Brasil, de 2011 a 2013, por medio de entrevistas semiestructuradas. Los datos fueron sistematizados en el software Atlas.ti y sometidos al análisis temático. Los aspectos que más contribuyen para aumentar las cargas de trabajo son la baja remuneración mensual, falta de recursos materiales, sobrecarga de trabajo, desgaste y enfermedad, relación con el usuario, y escasez de recursos humanos, prevaleciendo cargas psíquicas y fisiológicas. Adecuación de las condiciones y organización del trabajo se muestran primordiales para amenizar las cargas de trabajo en el escenario de la atención primaria a la salud, y para promover la salud, el bien-estar y la cualidad de vida en el ambiente de trabajo de los técnicos de enfermería.

Palabras clave cargas de trabajo; salud del trabajador; técnico de enfermería; atención primaria a la salud.

\section{Notas}

1 Universidade de Brasília, Departamento de Saúde Coletiva, Programa de Pós-graduação em Saúde Coletiva, Brasília, Distrito Federal, Brasil.

$<$ magda.scherer@pq.cnpq.br>

Correspondência: Universidade de Brasília, Núcleo de Estudos de Saúde Pública, SCLN 406, Bloco A, $2^{\circ}$ andar, Asa Norte, CEP 70847-510, Brasília, Distrito Federal, Brasil.

2 Universidade de Brasília, Brasília, Distrito Federal, Brasil.

$<$ alfangell@hotmail.com>

3 Universidade Federal de Santa Catarina, Centro de Ciências da Saúde, Departamento de Enfermagem, Florianópolis, Santa Catarina, Brasil.

<piresdp@yahoo.com>

4 Universidade do Estado de Santa Catarina, Centro de Educação Superior do Oeste, Chapecó, Santa Catarina, Brasil.

$<$ letrindade@hotmail.com>

5 Universidade Federal do Pará, Instituto de Ciências da Saúde, Faculdade de Enfermagem, Belém, Pará, Brasil.

$<$ sofiaresque@hotmail.com>

6 Fundação Oswaldo Cruz, Escola Politécnica de Saúde Joaquim Venâncio, Rio de Janeiro, Rio de Janeiro, Brasil.

$<$ monicavi@fiocruz.br> 


\section{Referências}

ALMEIDA, Paulo J. S.; PIRES, Denise E. P. O trabalho em emergência: entre o prazer e o sofrimento. Revista Eletrônica de Enfermagem, Goiânia, v. 9, n. 3, p. 617-629, 2007.

BARBOSA, Thiago L. A. et al. Expectativas e percepções dos estudantes do curso técnico em enfermagem com relação ao mercado de trabalho. Texto Contexto Enfermagem, Florianópolis, v. 20, n. esp., p. 45-51, 2011.

BERTONCINI, Judite H.; PIRES, Denise E. P.; SCHERER, Magda D. A. Condições de trabalho e renormalizações nas atividades das enfermeiras na saúde da família. Trabalho, Educação e Saúde, Rio de Janeiro, v. 9, Supl. 1, p. 157-173, 2011.

\section{CONSELHO REGIONAL DE ENFERMAGEM}

DA BAHIA. Estresse ocupacional do profissional de enfermagem, subdimensionamento de pessoal. Disponível em: <http://ba.corens. portalcofen.gov.br/wp-content/uploads/ 2014/09/ESTRESSE-OCUPACIONAL-DOPROFISSIONAL-DE-ENFERMAGEM.pdf $>$. Acesso em: 30 mar. 2015.

COTTA, Rosângela M. M. et al. Organização do trabalho e perfil dos profissionais do Programa Saúde da Família: um desafio na reestruturação da atenção básica em saúde. Epidemiologia e Serviços de Saúde, Brasília, v. 15, n. 3, p. 7-18, 2006.

CRUZ, Roberto M. et al. Saúde docente, condições e carga de trabalho. Revista Electrónica de Investigación y Docencia (REID), Jaén, v. 4, p. 147-160, 2010.

LIMA, Letícia et al. Satisfação e insatisfação no trabalho de profissionais na atenção básica. Escola de Enfermagem Anna Nery, Rio de Janeiro, v. 18, n. 1, p. 17-24, 2014.

DEJOURS, Christophe; ABDOUCHELI, Elisabeth; JAYET, Christian. Psicodinâmica do trabalho: contribuições da escola dejouriana à análise da relação prazer, sofrimento e trabalho. São Paulo: Atlas, 2011.
DILÉLIO, Alitéia S. et al. Prevalência de transtornos psiquiátricos menores em trabalhadores da atenção primária à saúde das regiões Sul e Nordeste do Brasil. Cadernos de Saúde Pública, Rio de Janeiro, v. 28, n. 3, p. $503-$ $-514,2012$.

ELIAS, Marisa A.; NAVARRO, Vera L. A relação entre o trabalho, a saúde e as condições de vida: negatividade e positividade no trabalho das profissionais de enfermagem de um hospital-escola. Revista Latino-Americana de Enfermagem, São Paulo, v. 14, n. 4, p. 517-525, 2006.

GEHRING JÚNIOR, Gilson et al. Absenteísmo-doença entre profissionais de enfermagem da rede básica do SUS Campinas. Revista Brasileira de Epidemiologia, São Paulo, v. 10, n. 3, p. 401-409, 2007.

GIRONDI, Juliana B. R. et al. Risco, vulnerabilidade e incapacidade: reflexões com um grupo de enfermeiras. Revista Eletrônica de Enfermagem, Goiânia, v. 12, n. 1, p. 20-27, 2010 .

KAISER, Dagmar E.; BIANCHI, Fabiana. A violência e os profissionais da saúde na atenção primária. Revista Gaúcha de Enfermagem, Porto Alegre, v. 29, n. 3, p. 362-366, 2008.

LAURELL, Asa C.; NORIEGA, Mariano. Processo de produção e saúde. São Paulo: Hucitec, 1989.

MAGALHÃES, Ana M. M.; RIBOLDI, Caren O.; DALL'AGNOL, Clarice M. Planejamento de recursos humanos de enfermagem: desafio para as lideranças. Revista Brasileira de Enfermagem, Brasília, v. 62, n. 4, p. 608-612, 2009.

MARTINS, Júlia T. et al. Significados de cargas de trabalho para enfermeiros de Pronto socorro/emergência. Ciência, Cuidado e Saúde, Maringá, v. 12, n. 1, p. 40-46, 2013.

MEDEIROS, Soraya M. et al. Condições de trabalho e enfermagem: a transversalidade 
do sofrimento no cotidiano. Revista Eletrônica de Enfermagem, Goiânia, v. 8, n. 2, p. 233$-240,2006$.

MININEL, Vivian A.; BAPTISTA, Patrícia C. P.; ANDRES, Vanda E. Cargas psíquicas e processos de desgaste em trabalhadores de enfermagem de hospitais universitários brasileiros. Revista Latino-Americana de Enfermagem, São Paulo, v. 19, n. 2, p. 340-347, 2011.

MORAES, Eliane P. Cargas de trabalho: um olhar sobre a estratégia saúde da família. 2013. 103 fl. Dissertação (Mestrado em Saúde Coletiva) - Faculdade de Ciências da Saúde, Universidade de Brasília, Brasília, 2013.

OLIVEIRA, Thays C.; CARVALHO, Liliane P.; SILVA, Marysia A. O enfermeiro na atenção à saúde sexual e reprodutiva dos adolescentes. Revista Brasileira de Enfermagem, Brasília, v. 61, n. 3, p.306-311, 2008.

RITTER, Renata S.; STUMM, Eniva M. F.; KIRCHER, Rosane M. Análise de burnout em profissionais de uma unidade de emergência de um hospital geral. Revista Eletrônica de Enfermagem, Goiânia, v. 11, n. 2, p. 236$-248,2009$.

ROBAZZI, Maria L. C. C. et al. Alterações na saúde decorrentes do excesso de trabalho entre trabalhadores da área de saúde. Revista Enfermagem UERJ, Rio de Janeiro, v. 20, n. 4, p. 526-532, 2012.

SANTANA, Leni L. et al. Cargas e desgastes de trabalho vivenciados entre trabalhadores de saúde em um hospital de ensino. Revista Gaúcha de Enfermagem, Porto Alegre, v. 34, n. 1, p. 64-70, 2013.
SANTINI, Stela M. L. et al. Perfil dos profissionais das equipes de saúde da família em municípios de pequeno porte de uma regional de saúde no Paraná e suas condições de trabalho. In: CONGRESSO CONSAD DE GESTÃO PÚBLICA, Brasília, 2010. Disponível em: <www.escoladegoverno.pr.gov. br/arquivos/File/Material_\%20CONSAD/ paineis_III_congresso_consad/painel_3/ Perfil_dos_profissionais_das_equipes_ de_saude_da_familia_em_municipios_de_ pequeno_porte_de_uma_regional_de_saude_ do_parana_e_suas_condicoes_de_trabalho. pdf>. Acesso em: 2 abr. 2014.

SCHERER, Magda D. A.; PIRES, Denise E. P.; SORATTO, J. O trabalho na Estratégia Saúde da Família. In: SOUSA, Maria F.; FRANCO, Marcos S.; MENDONÇA, Ana V. M. (orgs.) Saúde da família nos municípios brasileiros: Os reflexos dos 20 anos no espelho do futuro, Campinas: Saberes Editora, 2014. p. 521-571. Cap. IV.

SCHMOELLER, Roseli et al. Cargas de trabalho e condições de trabalho da enfermagem: revisão integrativa. Revista Gaúcha de Enfermagem, Porto Alegre, v. 32, n. 2, p. 368-377, 2011.

TRINDADE, Letícia L.; PIRES, Denise E. P. Implicações dos modelos assistenciais da atenção básica nas cargas de trabalho dos profissionais de saúde. Texto Contexto Enfermagem, Florianópolis, v. 22, n. 1, p. 36$-42,2013$.

Recebido em: 15/01/2015

Aprovado em: 30/03/2015 